\section{Mach mal Pause}

C. Hurni

"Mach mal Pause» - ein Werbespruch, wenn ich mich richtig entsinne. "Mach mal Pause" steht oft in Verbindung mit einem süssen Riegel. Er soll einem die Pause versüssen. Pausen brauchen also, gemäss Werbepsychologie, eine Art Belohnung, einen Lockvogel. Pausen sind süss, so will es die Werbung. Vielleicht nicht die Zahnärzte ... Obschon, lukrativ wären und sind sie ja auch für diese, und Pausen dienen - so habe ich den Eindruck - in der zivilisierten, erwachsenen Bevölkerungsgruppe hauptsächlich der Wirtschaftlichkeit. Im Kino gibt es in der Mitte des Films beispielsweise meistens eine Pause. Nicht, weil dort die Zuschauer unbedingt eine Pause nötig hätten, sondern damit der Kinokiosk seine Naschereien feilbieten kann. Keine Pausen zu machen hat je nachdem - ob für den gesunden oder den kranken Menschen - einen sehr hohen Preis.

Pausen haben seit dem Kranksein einen sehr hohen und wichtigen Stellenwert. Mein Körper will Pausen, verlangt nach ihnen, benötigt Ruhe. Gönne ich sie ihm nicht, rächt er sich meistens ziemlich prompt, und ich muss büssen. Die gehörigste Busse ist ein weiterer Schub, doch meistens verschlimmern sich einfach die bereits vorhandenen Symptome. Busse genug ... Im Grunde nicht nett von meinem Körper, mir so radikal Grenzen zu stecken, doch andererseits auch sehr vernünftig. "Mach mal Pause» mit oder ohne Riegel, das ist ihm egal. Er will einfach Pausen. Punkt.

Korrespondenz: Charlotte Hurni Forchstrasse 251 CH-8032 Zürich
Da ich krankheitshalber im Moment nicht arbeiten kann, ist genügend Zeit für Pausen vorhanden. Sie liessen sich also gut in den Tagesrhythmus einbauen. Nur, Pausieren ist nicht in erster Linie eine Frage der benötigten Zeit, sondern des benötigten Platzes und Raumes. Für mich eine ganz neue Erkenntnis. Wie gebe ich mir den Raum, den Platz? Ich, die ich - noch gesund - ruhe- und pausenlos umhergehetzt bin. Sobald es mir nur eine Spur besser geht, lasse ich die Pausen wieder links liegen. Das heisst, ich versuche es ... Nur, meine Krankheit weiss es besser und hat einen grossen Zeigefinger. Pausenfinger!

Allgemein stelle ich fest, in alte Verhaltensmuster zurückzufallen, sobald es mir gesundheitlich besser geht. Seit ich an MS erkrankt bin, gibt es eine sehr kranke, eine kranke und eine etwas gesündere Charlotte H. Entsprechend fallen die Pausen aus. Entsprechend verändert sich das "Bei-mir-selber-Sein". Eine Lebensqualität, die ich seit meiner Krankheit neu kennen und schätzen gelernt habe. Eine Qualität, die ich als Gesunde kaum kannte und viel zu wenig oder gar nicht pflegte. Ich hatte lange Jahre kaum «Pausenkulturn. Nachdem nun ein Jahr lang ein Schub dem letzten gefolgt ist, und ich ziemlich stillgelegt war, glaube ich, derzeit in eine gesündere Phase «hinüberzutreten». Meine Energie kehrt allmählich zurück, das Gehen geht langsam aber stetig besser, der Rücken schmerzt eine Spur weniger. Die Blasenund Sehstörungen schränken mich zeitweise noch ein. Doch immerhin, mein Körper scheint nun mir die ersehnte Pause zu gönnen; die lange ersehnte Erholung von einem Schub! Um so mehr gilt es jetzt wohl, weiterhin gut und klug mit meinen vorhandenen Kräften hauszuhalten. Mich nicht überzustrapazieren - im altbekannten Muster.

Eine gewisse Zeit lang erlebte und verstand ich die mir von meinem Körper so vehement auferlegten Forderungen nach Pausen als "Gefangenschaft". Doch vor ein paar Tagen realisierte ich, dass es sich eigentlich um eine ganz besondere Freiheit handelt. Die Freiheit der Kranken, Pausen machen zu müssen

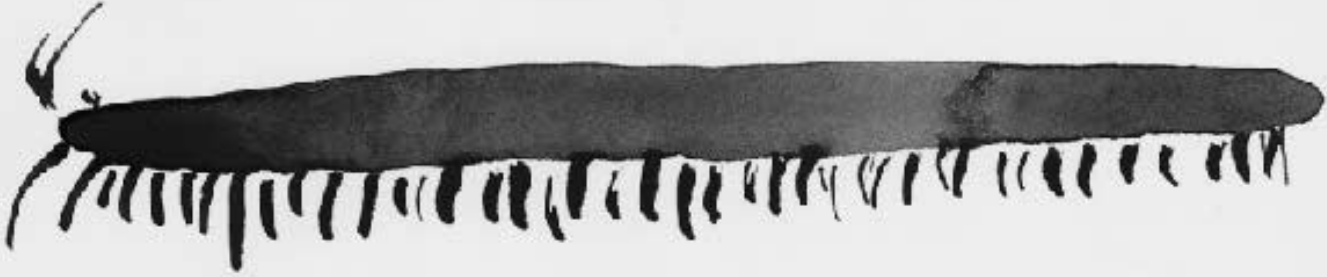


und zu dürfen. Eine etwas abstruse Freiheit, ich weiss. Und doch, wie bringe ich es fertig, diese Pausenfreiheit-Erkenntnis in meine gesünderen Phasen zu integrieren? Sozusagen als Pausenbrot, als süssen MS-Riegel, MilchSchokoladen-Riegel ... Welchen Werbespot soll ich für mich abspulen lassen? Ein Zuckerschlecken ist die Krankheit ja nun trotzdem weiterhin nicht! Immer sitzt die Angst vor der nächsten Attacke, vor dem nächsten Schub im Nacken.

\section{Pausen-Plätze}

Zum ersten Mal begegnete ich ihnen im Kindergarten. Dort gab es Platz für Pausen. Und auch später, während der ganzen Schul- und Ausbildungszeit waren Pausen selbstverständlich eingeplant und es gab den nötigen Platz und die Zeit dafür. Der süsse Riegel war zwar nicht so gerne gesehen, doch um so besser schmeckte er. Plötzlich erwachsen, blieb höchstens der Riegel, für Pausen fehlte (vermeintlich) die Zeit und der Platz. $\mathrm{Zu}$ viele Verpflichtungen und Projekte - oft selbstauferlegte - verbauten mir die Ruheoasen. Ich habe mir den Alltag mit Müssen, ewigem Tun und Schaffen und "am Riämä nä» zubetoniert.
Zurzeit bin ich wieder in einer Art Ausbildungsphase. Ich will und muss lernen, mit Krankheit, Einschränkung und - Pausen! - zu leben. Lernen, das Gelernte anzuwenden. In guten und in schlechten Zeiten. Die gute Mitte zwischen Aktivität und Ruhen finden. Pausen sind und bleiben jetzt nicht nur sehr wichtig, sondern sind gesellschaftlich gesehen sogar wieder legitimiert. Erwachsene kranke Menschen dürfen und müssen Pausen machen. Wirtschaftlichkeit spielt keine Rolle, so scheint mir. Ein Privileg gewissermassen, so wie man es als Kind und eben während der Schul- und Ausbildungszeit hat.

Nicht zu vergessen die "schöpferischen Pausen", aus welchen heraus ich schliesslich schreibe, male und eben - schöpfe.

Mein Körper als Lehrmeister, Ratgeber, Schöpfer und Pausenfinger. Ich will auf ihn hören: Mein Rücken schmerzt, die Müdigkeit macht sich breit und breiter, die Augen bringen den Text nur noch schwer "auf die Reihe». Der deutliche Wink für eine ausgiebige, wohltuende Pause.

Ich «mach' mal Pause»

\section{Von der Kunst, eine Lotosblüte zu sein}

\section{Erlebnisse einer werdenden Mutter}

\section{S. Rüster}

«Nun gehen wir alle in den Schneiderinnensitz!» sagt Asha sanft.

Ich muss kichern. Ich bin das erste Mal im Schwangeren-Yoga.

"Wir singen jetzt ein Mantra.»

Asha haucht ihre Worte bis in die letzte Reihe. Perfekt, so wie ihr nie endendes halbes BuddhaLächeln. Doch, das Lächeln kann enden. Das erfahre ich, als es an die Kursgebühren geht. Obwohl ich erst in der letzten Woche des Monates eingestiegen bin, will sie die 120.- DM für den ganzen Monat haben.

Korrespondenz:

Susanne Rüster

Matterhornstrasse 3

D-14129 Berlin
Ist sie aber nicht beim Kassieren, schwebt Asha, die Zierliche, uns davon. Alle Frauen sollen eine sich im Wind neigende Lotosblüte sein und ihre schweren Bäuche vergessen. Im Schneiderinnensitz schlafen meine Beine ein und ich bin erleichtert, als es in den Vierfüsslerinnenstand geht.

So ist das: Seit jenem Tag, als der Schwangerschaftstest den roten Punkt gezeigt hat, bin ich eingetaucht in eine fremde Welt. Es beginnt damit, dass ich einen Ratgeber für Schwangerschaft und Geburt kaufen will. Zurück komme ich mit: Das andere Babybuch, Baby-1x1, Schwanger in Berlin, Mutter und Kind, Berlin rund ums Baby und schliesslich Das Leben mit einem Neugeborenen (ein Gegenleitfaden, was mich besonders neugierig macht). Dabei wollte ich eigentlich nur wissen, was in meinem Körper jetzt geschieht. Was soll ich essen, welcher Sport ist erlaubt, welche Probleme gibt es in der Schwangerschaft? Ich staune: Ich soll einen Geburtsplan schreiben und ihn dem Klinikpersonal aushändigen. Wie ich mir meine Geburt vorstelle. Meine Vorstellung von der Geburt ist vage und ängstlich. Aber im Krankenhaus würde man schon wissen, was zu tun sei. Im Krankenhaus ... Aber in welchem? Schon wieder überrascht mich der Ratgeber. Heutzutage geht eine Frau nicht mehr einfach in die nächstgelegene Entbindungsklinik. Soll sie überhaupt eine Klinik aufsuchen oder in ein Geburtshaus gehen oder in eine 
gynäkologische Praxis für ambulante Geburten, oder soll sie ambulant bei einer freiberuflich praktizierenden Hebamme entbinden oder gar zu Hause?

Ich gehe auf Schwangerschaftstourismus. Die Kliniken buhlen um die werdenden Mütter. Flugs bin ich von der Patientin befördert zur umworbenen Geldgeberin. Im Krankenhaus spricht der Chefarzt über die natürliche Geburt, die auf Wunsch der Mütter angeboten werde. Man betreibt ja Dienstleistungsmedizin. Immer vorausgesetzt, es sei nicht eine kontrollierte Geburt erforderlich. Nicht ganz klar wird mir, wie sich die natürliche Geburt unterscheidet von einer normalen Geburt, wo doch jede Geburt ein natürlicher Vorgang ist. Beim Chefarzt wird selbst der Kaiserschnitt zum Spaziergang. Betäuben, aufschneiden, Kind holen, wieder zunähen. Maximal halbe Stunde. Hier erlebe ich es zum ersten Mal: Ich bin ahnungslos. Denn kaum hält der Chefarzt inne, ziehen die jungen Mütter (und Väter) ihre Spickzettel und fragen $a b$ mit ernster, wichtiger Miene.

"Wird routinemässig rasiert?»

"Welche Kaiserschnittrate haben Sie?»

"Führen Sie Wassergeburten durch?»

"Wie hoch ist Ihre Dammschnittquote?"

Während des anschliessenden Vortrags über Neonatalogie (alle im Publikum scheinen zu wissen, was das ist) beschliesse ich, doch in die nächstgelegene Entbindungsklinik zu gehen. Der Vortrag über die Neugeborenenmedizin beginnt mit einer Anekdote aus dem Kreissaal:

"Jetzt hecheln", befiehlt die Hebamme.

"Was is'n das?!"

"Na, wie ein Hund machen!»

"Wau! Wau!"

Doch schon zeigt die Leinwand winzige, faltige Babys in Brutkästen, verdrahtet, mit Sonden gespickt, inmitten von ausschlagenden Zeigern und zuckenden Kurven. Ab der 25. Woche besteht eine Überlebenschance von etwa 30\%, sagt die Neonatalogin, ab der 30 . Woche schon von $90 \%$. Ich habe jetzt 34 Wochen und verlasse die Uniklinik fluchtartig. Trotzdem finden sich in meiner Tasche Kursangebote für Schwangerschaftsgymnastik, Geburtsvorbereitung und Säuglingspflege, ein Anmeldeformular für das Prager Eltern-Kind-Programm und eins für das Baby-Schwimmen.

Warum darf ich eigentlich nicht eine ganz normale Geburt haben?! Einfach in eine Entbindungsklinik gehen, und die Entscheidung, was während der Geburt (Asha sagt: unter der Geburt) zu tun ist, dem medizinisch geschulten Personal überlassen? Beim Schwangeren-Yoga habe ich auch immer noch Schwierigkeiten, allen Menschen, Tieren und Pflanzen auf der Welt die besten Wünsche zu übermitteln. Sat nam. Wenn wir unser Mantra singen sollen, summe ich Victoriih-aa-ha. Dank der Fruchtwasseruntersuchung weiss ich, dass es ein Mädchen wird. Das mögen die Leitfäden nun gar nicht. Hoffentlich hat sich Victoria bei diesem Eingriff der Apparatemedizin nicht zu sehr erschreckt! Und so bemühe ich mich sehr, gegen meine im Schneiderinnensitz einschlafenden Beine anzudenken - weit und locker wer- den! Auch versuche ich, die weite Grätsche auf dem Fussboden wenigstens so lange auszuhalten, bis ich den Leitartikel zu Ende gelesen habe. Dann kommt das Schlimmste: Die Hocke. Gebären geht am besten in der entspannten Hocke, hat Asha, die Dunkeläugige gesagt. Die Knie auseinander, die Fersen fest am Boden, so schaffe ich leider nur ganz kurze Artikel und weiss hinterher nicht, was ich gelesen habe. Doch Asha hält unerbittlich an dieser Wehenübung fest. Die hat nichts mit Wehen zu tun. Sie soll uns aber ein Gefühl für Schmerz vermitteln und danach die schöne Gewissheit, dass wir mit Schmerz auch ohne Pille umgehen können. Ein bisschen Entspannungsmusik, Massageöl und kraftvolle Gedanken. Den Schmerz der entspannten Hocke ertrage ich nur sieben Minuten und auch die Aussicht, die entspannte Hocke später neben meinem Kind im Buddelkasten zur Perfektion zu bringen, hilft nicht gegen den Druck in den geschwollenen Beinen. Und was ist, wenn bei der Geburt keine genügend gelenkige Hebamme Dienst hat, die auf dem Boden liegend, das Kind entgegennimmt?! Aber die homöopathischen Kügelchen für eine leichte Geburt nehme ich weiterhin. Auch schadet es sicher nicht, ganz tief in den Bauch zu atmen. Und so wandere ich viel - wie ich es schon immer tat - und blase mich dabei auf und atme pfthhh - ganz lang wieder aus. Dabei zähle ich die Schritte, denn ausatmen soll ich länger als einatmen, um eine Hyperventilation zu vermeiden. Beim Aufblasen fühle ich mich jetzt richtig dick, und wenn wir beim Yoga wieder Lotosblüte sein sollen, döse ich vor mich hin. Das bekommt mir sehr gut.

Der wohlsortierte Klinikkoffer ist eine Wissenschaft für sich. Die Einkaufsliste in meinem Geburtsvorbereitungsbuch umfasst zwei Seiten. Immer wieder taucht die Eigenschaft kochfest auf. Herausreissen will ich die Liste nicht, das schwere Buch mitnehmen auch nicht. Kühn verlasse ich mich auf meine Intuition. Ich kaufe ein par winzige Flügelhemdchen mit Bändchen. Wie mögen die wohl nach der Wäsche aussehen?! Aber die Bezeichnung Erstlingshemdchen ist so rührend. Dass dagegen die einteilige Babywäsche als Body bezeichnet wird! Darunter stelle ich mir ein erotisches Wäschestück für die ausgewachsene Frau vor. Das Erstlingshemdchen kostet 6.50 DM, das Nachthemd für die junge Mutter, vorn zu knöpfen, dagegen 49,50 DM, ebensoviel wie der Stillbüstenhalter Anita.

"Ein schön ausgewaschenes Hemd tut's doch auch", sagt mein Mann und holt flugs ein paar alte Oberhemden aus der Kleiderspende.

Und ehe ich 49,50 DM für einen Stillbüstenhalter ausgebe, selbst wenn er kochfest ist, will ich erst einmal sehen, wie das mit dem Stillen überhaupt geht. Meine kleinen Siege über die Gebote der Geburtsvorbereitungsgurus: Erst mal abwarten! Und nicht beunruhigt sein, wenn die Fachfrauenliteratur von der aus Milchpulver anzurührenden Säuglingsmilch abfällig als künstliche Milch spricht und deren Hersteller mit solch einer Abscheu die Vertreiber von Säuglingsnahrung nennt, dass die Bezeichnung Verbrecher naheliegt. 
Stillen soll doch Freude machen. Ich kenne eine junge Mutter, die sich seit der Geburt von Laurenz seit nunmehr fünf Monaten mit Stillhütchen und der Milchpumpe herumplagt und täglich mehrere Stunden mit der Nahrungsbeschaffung für ein bleiches, mageres Baby zu tun hat. Seit der Geburt hat sie nie mehr ausgeschlafen und ist halb verrückt vor Schlafmangel und von der strengen Diät, die ihr Laurenz ihr abverlangt. Bei Orangen, Tomaten, Zwiebeln wird der Babypo wund. Bei Gemüse, Obst, Brot bekommt Laurenz Blähungen. Kaffee lässt ihn noch mehr nach der Mutterbrust schreien und Alkohol würde ihn dumm machen. Ich freue mich ja jetzt schon auf das Glas Champagner alsbald nach der Geburt. Ja, wenn das mit dem Stillen nicht zu unser beider Freude klappen sollte, dann werde ich ausprobieren, ob Victoria gegen künstliche Milch tatsächlich Allergien entwickelt. Mein Mann schüttelt dazu den Kopf.

«Ich weiss es einfach, dass Victoria ein vernünftiges Kind wird!"

Ich entscheide mich auch gleich gegen das Erlernen dreier verschiedener Techniken für das Wickeln von Stoffwindeln. Victoria wird ein vernünftiges Kind und ihr Po wird robust genug für PampasWindeln sein! Einen Säuglingspflegekurs brauche ich also nicht mehr. Dafür habe ich Zeit, noch alle Kinofilme meiner Wunschliste anzusehen.

Die klassische Geburt kündigt sich mit Vorwehen an und dem sicheren Gefühl, dass gleich etwas Grosses geschieht, hat Asha gesagt. Victoria kündigt sich dagegen mit einem schmerzlosen, vorzeitigen Blasensprung an. Meine energischen Ratgeber für eine natürliche Geburt haben zum Glück ein Schlusskapitel andersartigen Verläufen gewidmet: den Komplikationen. Auch dann soll ich an meinem Geburtsplan festhalten. Ehe ich aber nach einem Gebärhocker fragen kann, hat mich die Ärztin schon ins Bett verfrachtet. Meine halbherzige Bitte, noch ein wenig herumzulaufen und die verschiedenen Haltungen für eine natürliche Geburt wenigstens einmal richtig auszuprobieren, lehnt sie ebenso ab wie das Wippen auf einem Pezziball. Die Badewanne für Wassergeburten erwähne ich gar nicht.

«Darf ich bei einer Wassergeburt mit in die Badewanne?»

Das war die Frage eines jungen, werdenden Vaters bei der Besichtigung dieser Klinik. Tatsächlich scheint die Badewanne nur Alibizwecken zu dienen - man betreibt ja Dienstleistungsmedizin - oder bei ganz renitenten werdenden Müttern zum Einsatz zu kommen. Der diensttuenden Ärztin ist auch egal, dass mein Mann nicht beim Geburtsvorbereitungskurs war ("Ich bin doch kein alternativer Spinner!») und daher nicht weiss, wie er sich hinter mich $\mathrm{zu}$ hocken und mich abzustützen hat
«Sie kommen sowieso an den Tropf!» Zumindest siezt sie mich.

Das Papier, das aus dem Wehenschreiber kommt, zeigt sehr sanfte Wellen. Ich weiss, das Schlimmste droht: die medizinisch überwachte Geburt. Ich lausche in mich hinein. Warum will Victoria nicht raus?! Ihr Herzchen schlägt quicklebendig, das zeigt die andere steilzackige Kurve, die sich neben der Wehenkurve ausnimmt wie die Dolomiten gegenüber Wanderdünen. Die nächste Zeit beobachte ich das Papier. Lang und länger quillt es aus dem Gerät und die ewigen Wellenlinien machen mich müde. Ich wäre eingeschlafen, wenn nicht von nebenan ein klagender Ton zu mir gedrungen wäre. Das ist es wohl, was Asha im Schwangeren-Yoga als Tönen bezeichnet hat: Schmerz in Ton umsetzen ohne aus dem Atemrhythmus zu geraten. Wie gern hätte ich jetzt Musikkassetten! Dieses Tönen ist wie eine Anklage. Doch die Kassetten sind in meiner Tasche und die steht irgendwo anders. Nicht umsonst empfiehlt ja «Der Klinik-Koffer", zwei Taschen zu packen; eine kleine für den Kreisssaal (Massageöl, Entspannungsmusik, Duftkerzen und Wollsocken) und eine grössere für hinterher. So bleibt nichts als der Blick auf die wellenbeschriftete Papierschlange.

Aufgerissene, tiefblaue Augen blicken mich an. Wo bin ich denn jetzt gelandet?! So ein wacher, erstaunter Blick! Dabei ist Victoria mit künstlichen Mitteln ans Licht der Welt getrieben worden und hat zu alledem noch eine Mutter, die die Einflüsterungen Ashas einfach vergessen hat. Ich habe nicht an die sich im Wind wiegende Lotosblüte gedacht und auch nicht ja-jaa-jaaa! gesagt, geschrien. Ja, was tut die werdende Mutter stattdessen?! Sagt «Nein, nei-ein!» Will die Peridural-Anästhesie. Der Ausweg der Feigen. Und das trotz zwanzig Stunden SchwangerenYoga zu 600.- DM!

"Jetzt hast du es geschafft!" sage ich zum nassen, zappelnden Wesen, das zum ersten Mal die Luft einzieht. Eine winzige Hand krallt sich fest, ein Mäulchen versucht sich durchs Hemd zu bohren. «Wir werden es gut haben miteinander». Gucken und Staunen. Victoria hat hellbraune verklebte Haare, ein Stumpfnäschen, zarte Augenbrauen, ein rundes Gesichtchen, hell und fein. Ja, wir haben es gut gemacht. Wir haben es einfach auf uns zukommen lassen. Gemeinsam verlassen wir den Kreisssaal. Das Kind streckt das Händchen hoch.

"Jetzt hat sie sich vom Himmel verabschiedet!» sagt die Hebamme. 